\title{
ESTADO POBLACIONAL DEL ALMENDRO COMO INDICADOR DE LA DISPONIBILIDAD DE HÁBITAT DE LAPA VERDE EN EL SURESTE DE NICARAGUA
}

Armando J. Dans ${ }^{[1]}$ Gladys Luna ${ }^{[2]}$ Christopher A. Jordan ${ }^{[3]}$

\section{Resumen}

La investigación se realizó en siete comunidades del Sureste de Nicaragua, con el objetivo de determinar el estado actual de la población de almendro (Dipteryx panamensis), como indicador del hábitat potencial para la lapa verde (Ara ambiguus). Se muestrearon 21 parcelas de 1 ha cada una, estableciendo tres parcelas por comunidad, también se entrevistaron a pobladores para determinar la relación de éstos con las especies en estudio. En las parcelas muestreadas, se contabilizaron un total de 93 árboles de almendro con Diámetro a la Altura del Pecho (DAP's) $\geq 70 \mathrm{~cm}$, lo que representa una densidad promedio de 4.4 almendros/ha. También, se identificaron posibles amenazas para las especies en estudio y su particularidad con las comunidades y grupos étnicos. Resultados de Modelos Lineales Generalizados (GLM) y análisis de correlación, indican que en la zona de estudio, no es el tamaño o diámetro de los almendros lo que mantiene poblaciones de lapas, sino que influyen mucho la densidad de individuos con DAP $\geq 70 \mathrm{~cm}$, el nivel de aislamiento de la comunidad, y la cantidad y tipo de usos que le dan los pobladores al árbol. Desde los modelos de Maxent, en los alrededores de 5 comunidades (La Bijagua, Bartola, Indian River, Corn River y Point of Rock) presentan condiciones viables para la distribución del hábitat potencial del almendro y por ende de lapas verdes.

Palabras clave: Almendro (Dipteryx panamensis); Lapa Verde (Ara ambiguus); hábitat; distribución espacial; uso tradicional; sureste de Nicaragua.

\section{Summary}

The research was done in seven communities in the southeast of Nicaragua, in order to determine the current condition of almond-tree population (Dipteryx panamensis), as an indicator of potential habitat for the Green Macaw (Ara ambiguus). 21 plots of 1 ha each were sampled, by establishing three plots per community. Community people were also interviewed to determine their relationship with the species object

\footnotetext{
[1] Ingeniero Agroforestal. arjadans@gmail.com.

[2] MSc, Recursos Naturales y Desarrollo Rural. Ecóloga. Docente de tiempo completo. esmeldaluna@gmail.com

[3] Candidato de Doctorado en Conservación de Vida Silvestre. Michigan State University
} 
of study. In the sampled plots, a total of 93 almond-trees were counted with Diameter at Breast Height (DBH's) $\geq 70 \mathrm{~cm}$, representing an average density of 4.4 almondtrees/ha. Potential threats were also identified to the species subject of study and its particularity with the communities and ethnic groups.

The results of Generalized Linear Models (GLM) and the analysis of correlation, indicate that in the study area, it is not the size or diameter of the almond trees that maintains the green macaws populations, but what influences much is the density of individuals with $\mathrm{DAP} \geq 70 \mathrm{~cm}$, the level of isolation of the community and the amount and type of uses that the community people gives to the tree. According to Maxent models, on the surroundings of 5 communities (La Bijagua, Bartolo, Indian River, Corn River and Point of Rock) it offers viable conditions for the distribution of potential habitat for almond-tree and thus, for green macaws.

Keywords: Dipteryx panamensis; Ara ambiguus; habitat; spatial distribution; traditional use; southeast of Nicaragua.

\section{Introducción}

El árbol de almendro es una especie clave para la fauna silvestre. Hasta 60 especies de aves, mamíferos e insectos, destacando tucán (Ramphastos sulfuratus), oropéndola (Psarocolius montezuma), mono (Cebus Capucinus) y zorro (Didelphis marsupialis), se alimentan de sus frutos y semillas. La lapa verde, además, anida entre sus ramas (Sánchez, PE; Flores, EM. 1992.)

En Nicaragua el rango de distribución del almendro se ha reducido con el avance de la frontera agrícola (Ruíz, et al., 2005). Con el inicio de la ganadería, la población de almendro empezó a descender al utilizarse en construcciones pesadas y para la elaboración de carbón (Riverstone, 2008).

Actualmente es poco frecuente encontrar almendros en estado natural, lo que repercute directamente en el estado poblacional de todas las especies que lo ocupan, entre ellas la lapa verde, especie clave para su sobrevivencia y el mantenimiento de sus poblaciones; por eso no es de extrañar que estas dos especies (almendro-lapa verde) presenten condiciones similares en Nicaragua, ambas se encuentran en peligro de extinción y a la vez enfrentan la reducción de sus hábitats. El almendro se encuentra protegido en Nicaragua bajo el sistema de vedas indefinidas ${ }^{[4]}$ y ambas especies se encuentran en los apéndices del CITES ${ }^{[5]}$, I (lapa verde) y III (almendro).

[4] Resolución Ministerial No. 029-2006-MARENA

[5] The Convention on International Trade in Endangered Species of Wild Fauna and Flora (Convención sobre el Comercio Internacional de Especies Amenazadas de Fauna y Flora Silvestres 
Esta investigación aporta al conocimiento ecológico de ambas especies, ya que el objetivo fue determinar y evaluar el estado poblacional y/o situación del almendro como especie que alberga la lapa verde en las comunidades del sureste de Nicaragua.

\section{Revisión de literatura}

En esta investigación el estado poblacional del Almendro es considerado, como la valoración y evaluación de todos aquellos factores y/o variables que pueden generar algún efecto en su población.

Se han considerado únicamente aquellos almendros que tienen potencial para albergar y dar alimento a la lapa verde (Ruiz, et al. 2005), plantean que un árbol portador (almendro), es aquel que tiene un diámetro mayor a los $70 \mathrm{~cm}$, ya que aportará material y alimento para la especie (Ara Ambiguus) o el mantenimiento de la biodiversidad. Tomando en cuenta este criterio se escogió como muestra, únicamente almendros con diámetros mayores o iguales a $70 \mathrm{~cm}$.

Stiles y Skutch (1995), apuntan que la lapa verde habita el dosel de los bosques húmedos de bajura y se alimenta preferentemente de los frutos de almendro de bosques densos. Vuelan largas distancias para alimentarse de almendros en potreros y áreas semi-abiertas.

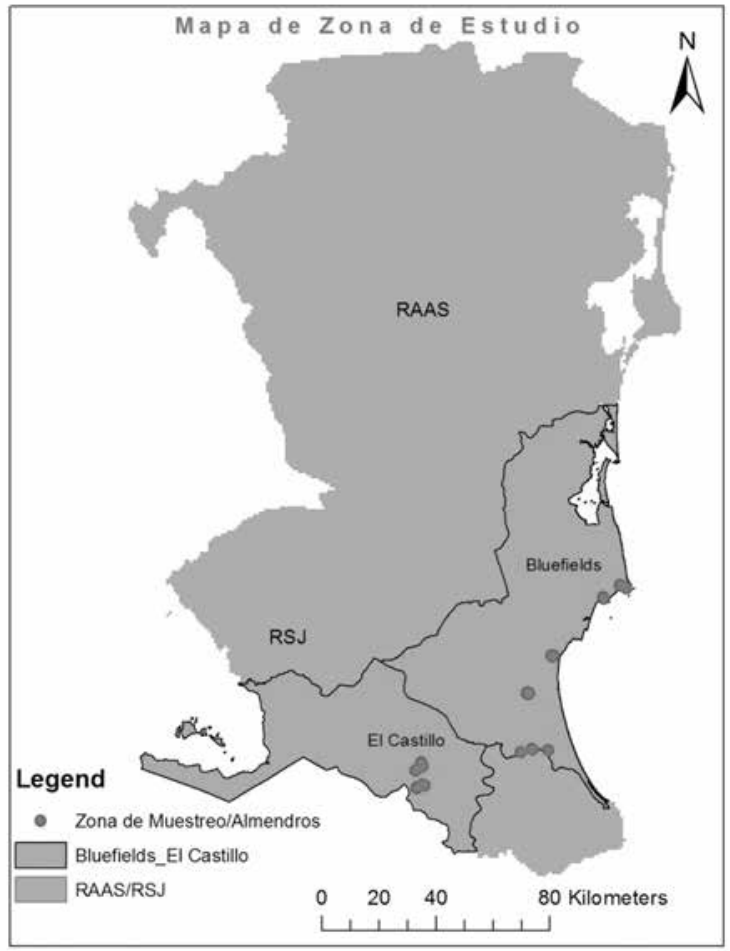

Figura No. 1. Zona de estudio/muestreo.
Madriz (2004), concluye que existe una relación de dependencia directa entre el árbol de almendro y la lapa verde en la zona norte de Costa Rica, registrando que el $87 \%$ de la dieta alimenticia de la lapa verde es de semillas de almendro y que el $80 \%$ de sus nidos son colocados en cavidades naturales de esta especie; incluso anota que existe una correlación entre la disminución del número de individuos de lapa verde y la pérdida de almendros en los remanentes boscosos y en potreros. 


\section{Materiales y métodos}

El estudio se realizó en siete comunidades del sureste de Nicaragua, dos en el departamento de Río San Juan (La Bijagua [N11 ${ }^{\circ} 03.455^{\prime}$ Wo84 ${ }^{\circ} 18.796^{\prime}$ ] y Bartola [N10 $59.087^{\prime}$ Wo84 $\left.{ }^{\circ} 18.589^{\prime}\right]$ ) y cinco comunidades en la Región Autónoma del Caribe Sur, las cuales

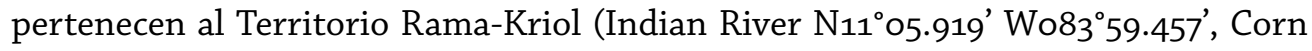

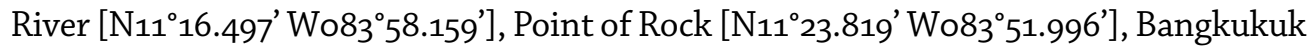
$\left[\mathrm{N}_{11}{ }^{\circ} 33.931^{\prime}\right.$ Wo $83^{\circ} 43.824^{\prime}$ ] y Monkey Point [N11 ${ }^{\circ} 35.669^{\prime}$ Wo83 $\left.39.505^{\prime}\right]$.

La muestra fue de 21 parcelas cuadradas que cubrían un área de $1 \mathrm{Ha}$ (10om $\mathrm{x}$ 10om) cada una. Para la selección de los lugares para establecer las parcelas se tomó en cuenta la opinión de los comunitarios del área de estudio y la elección de zonas potenciales para la alimentación y/o anidamiento de las lapas verdes, por tanto sólo se muestrearon árboles con DAP $\geq 70 \mathrm{~cm}$.

Dentro de cada parcela se contabilizó los almendros, se comprobó que su DAP fuese $\geq 70 \mathrm{~cm}$ y se geo-referenció. Para el avistamiento de lapas verdes o de nidos activos dentro de la parcela, se realizó observaciones durante todo el día.

Se aplicaron 67 entrevistas a comunitarios, con la intención de diagnosticar el factor antropogénico que puede influenciar el estado poblacional del almendro como hábitat potencial para la lapa verde.

Se corrieron Modelos Lineales Generalizados (GLM) y se hizo correlaciones utilizando los programas estadísticos Jump (JMP 9.0, 2010) y Rstudio (3.0.2, 2013), con el objeto de analizar la dependencia de una variable respuesta (número de árboles por hectárea) ante distintas covariables (aislamiento, cantidad de usos del almendro, sin usos del almendro, DAP). Estos modelos intentan explicar la variación de las densidades de almendro entre las comunidades y la posible influencia de otras variables ante tal variación.

Con los registros de almendros georeferenciados en las parcelas, se corrieron modelos de máxima entropía en el programa Maxent (3.3.3k, 2011), cuyo propósito es estimar distribuciones de probabilidad de máxima entropía sujeto a restricciones dadas por la información ambiental (Phillips et al, 2006), para conocer la distribución del hábitat viable de la especie Dipteryx panamensis en la zona de estudio para individuos con DAPs $\geq 70 \mathrm{~cm}$, Maxent utiliza los registros geográficos de los almendros muestreados y la información de las capas bioclimáticas y a partir de ello define el "hábitat ideal" para la especie. Compara ese hábitat ideal con las condiciones en las otras zonas del mapa donde no se hizo el muestreo, para indicar zonas que presentan condiciones similares a los puntos georeferenciados. 
Primero se elaboró una capa recortada de las zonas de estudio (Región Autónoma del Caribe Sur RACS y Rio San Juan RSJ) en ArcGis (10.1, 2012); luego se descargaron capas geográficas de diferentes variables bioclimáticas (precipitación, temperatura, áreas protegidas, huella humana) en formato Raster; se recortaron por máscara para adaptarlas al tamaño de la zona de estudio (RAAS/RSJ) y se convirtieron a formato ASCII, para correr los modelos en Maxent. Con los resultados del modelo se elaboraron mapas en ArcGis, para ilustrar la distribución del hábitat potencial del almendro en la zona de estudio.

\section{Resultados y discusión}

\section{- Población de almendros potenciales para hábitat de lapa verde}

En las 21 has muestreadas, en las siete comunidades se registró un total de 93 almendros con DAP $\geq 70 \mathrm{~cm}$, lo que representa una densidad promedio de 4.4 árboles/ha, con un DAP promedio de 114.4cm (figura 2).
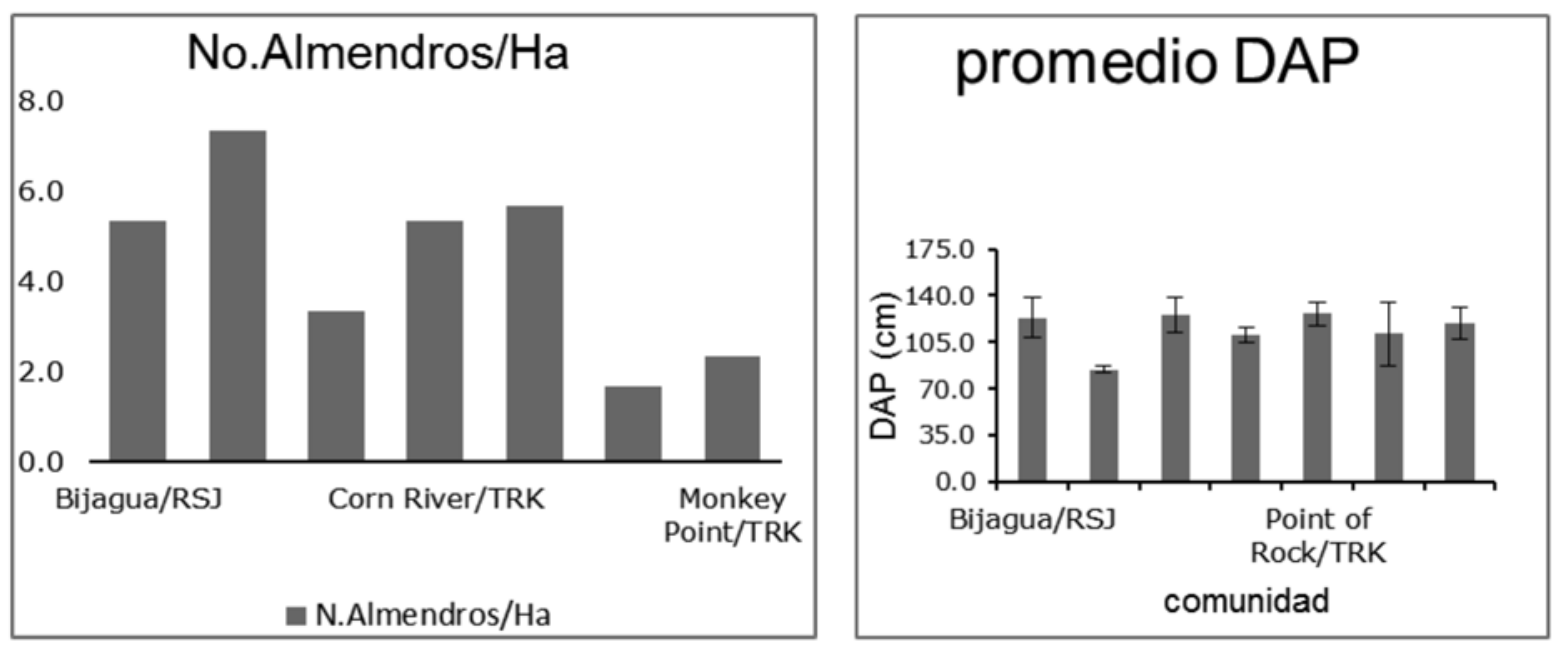

Figura No. 2. Densidad poblacional (número de individuos por hectárea) por comunidad (izquierda), y promedio de DAP por comunidad (derecha) [las barras representan el error estándar].

Es notable la variación en las densidades de almendro entre las comunidades (figura 2, izquierda), mientras que los promedios diamétricos (derecha) se comportan de forma más homogénea entre las mismas; lo que indica que es común encontrar almendros con diámetros cerca de la media $(114.4 \mathrm{~cm})$ en las comunidades de estudio.

La comunidad Bartola sobresale de todas las comunidades respecto a la densidad de almendros por hectárea, además ésta misma comunidad es la que tiene los almendros con menor DAP con un promedio de $84.5 \mathrm{~cm}$, lo que podría indicar que esta 
comunidad presenta una alta tasa de sobrevivencia post-germinación del almendro, en consecuencia podría decirse que es de las comunidades con mayor potencial para el hábitat de la lapa verde.

glm(formula $=$ N.almendros_Ha $\sim$ N.usos + no.lo.utilizan + promedio.DAP + Distancia)

Coefficients:

Estimate Std. Error t value $\operatorname{Pr}(>|\mathrm{t}|)$

(Intercept) $\quad 4.292349 \quad 1.349179 \quad 3.181 \quad 0.00229$ **

N.usos $\quad-0.483518 \quad 0.157838 \quad-3.063 \quad 0.00324$ **

Tres de las covariables utilizadas en el modelo lineal generalizado (GLM) resultan altamente significativas (N.usos $\mathrm{p}=0.00324$, promedio.DAP $\mathrm{p}=0.00376$, Distancia $\mathrm{p}=2.34 \mathrm{e}-15$ ) (figura No. 3). (Cuadro No.1).

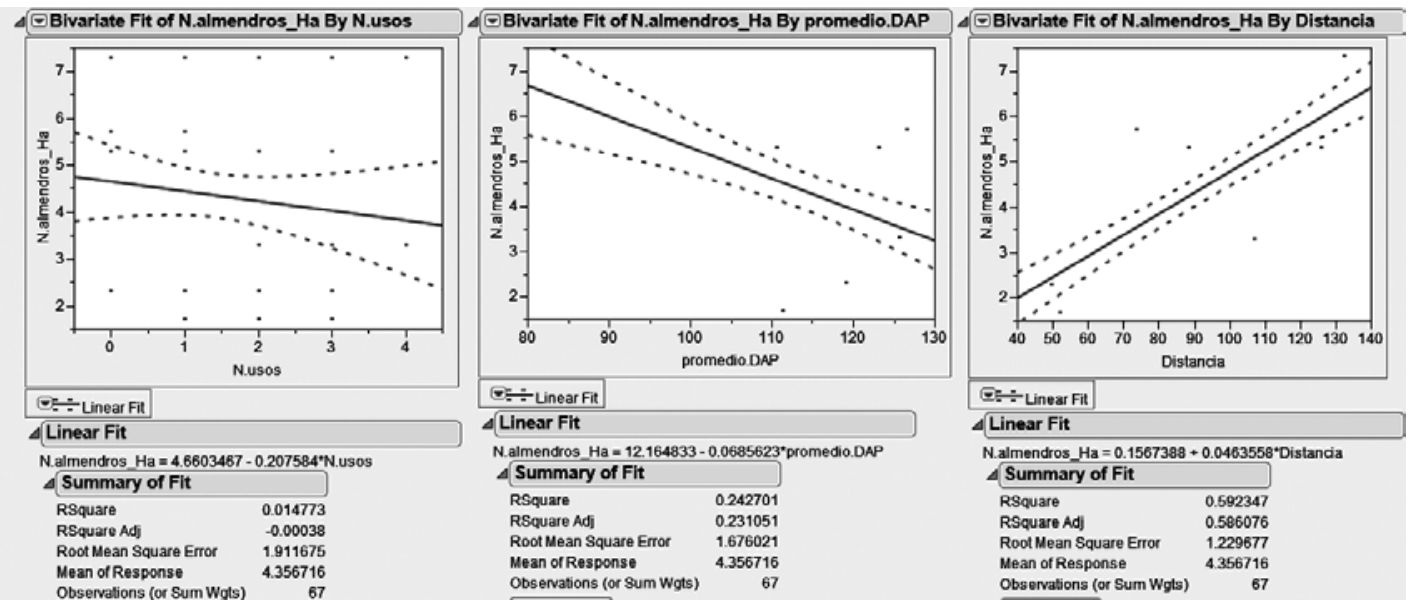

Figura No. 3. Correlación entre la densidad de Almendros vs el número de usos del almendro (izquierda), DAPs (centro), Aislamiento (derecha).

La primera correlación (número de usos versus densidad de almendro) indica que entre menos uso le dan los pobladores al almendro, es probable encontrar densidades más altas en los alrededores de las comunidades. A simple vista esto podría parecer lógico, y en cierta medida lo es; sin embargo, las observaciones y entrevistas indican que el contexto del uso del almendro es más complejo. 
En las comunidades del territorio Rama y Kriol hacen un uso tradicional de la especie, más apegado al fruto y semilla, como por ejemplo la elaboración de pozol (Ibo buña) entre otras bebidas y productos comestibles. Estos usos, si bien podría tener un efecto en la regeneración de los almendros, es poco probable que afecten la densidad a nivel de paisaje. Sin embargo, en las últimas décadas, el avance de colonos ha perjudicado, al utilizar mayores cantidades de almendro para leña, madera y carbón.

En las comunidades de El Castillo, los pobladores no usan el fruto de almendro, sino que usan el árbol para leña y madera en construcciones agrícolas (puentes, corrales y postería). Los usos que le dan los mestizos al almendro, probablemente afectan más las densidades de esta especie, que los usos que hacen las comunidades indígenas. Así, la correlación negativa entre el número de usos y la densidad de almendros, puede indicar que la manera mestiza de utilizar la especie, está superando los usos tradicionales. Sin embargo, no se cuenta con datos suficientes para comprobar esta hipótesis, siendo recomendable estudiar la regeneración y densidades de la especie Dipteryx panamensis en relación a los usos tradicionales y no tradicionales que le dan en las comunidades en estudio.

En cuanto a las densidades de almendro y el promedio de sus diámetros, se encontró que a mayor densidad existe menor promedio de DAP, lo cual confirma que el comportamiento en Bartola es patrón general para la zona de estudio. Aparentemente la población de almendros en Bartola se está regenerando exitosamente en términos del tamaño poblacional, lo que confiere a este sitio gran relevancia para los programas de conservación de lapa verde y de almendro a nivel nacional.

Los resultados del modelo lineal generalizado indica que la relación que existe entre las densidades de almendro y las temporadas de avistamientos de lapas verdes es significativa ( $\mathrm{p}=0.00149$ ), es decir que entre más almendros existen cerca de las comunidades es más probable que se observen lapas verdes durante todo el año en los alrededores de tales comunidades, confirmando con ello que el almendro es una especie clave para especies silvestres como la lapa verde, tal como exponen Sánchez y Flores (1992.).

La correlación entre las densidades de almendro y la covariable de aislamiento, la cual toma como referencia los cascos urbanos más cercanos (San Carlos y Bluefields), indican que, entre más aisladas estén las poblaciones de almendro es más probable encontrar densidades altas de estos. Esto quizás podría explicar las bajas densidades de almendro en las comunidades de Bangkukuk y Monkey Point, las cuales están más cerca de la ciudad de Bluefields, ciudad que representa un mercado potencial para los productos de almendro (pozol, carbón, madera). 
De igual forma, al analizar la dependencia de las temporadas de avistamiento ante el nivel de aislamiento de las comunidades, el GLM resultó muy significativo ( $\mathrm{p}=1.00 \mathrm{e}-07$ ), es decir que entre más aisladas estén las comunidades es más probable observar lapas verdes durante todo el año en las comunidades.

\section{- Modelos de distribución de hábitat potencial para almendros en el sureste de Nicaragua}

Se corrieron modelos de distribución de hábitat potencial para observar las áreas donde Dipteryx panamensis puede desarrollar poblaciones viables en la zona de estudio (RACS/RSJ). A continuación los resultados:

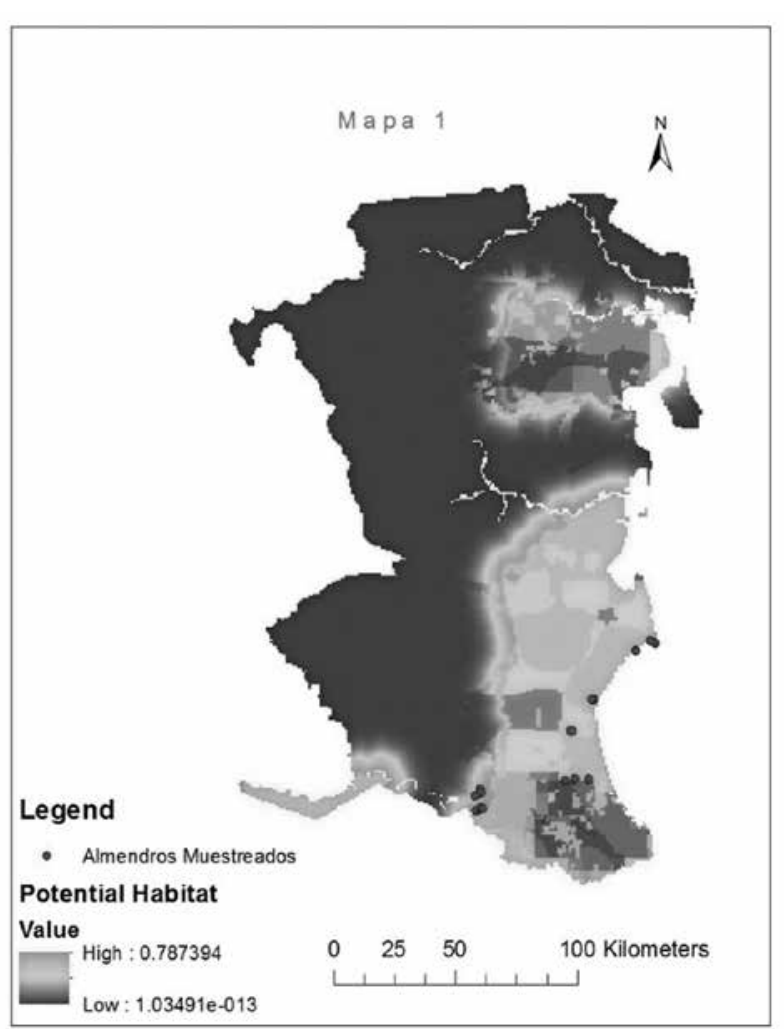

Figura No. 4. Distribución del hábitad potencial del almendro, tomando variables de área protegida y huella humana.

\section{Modelo 1}

En este modelo se utilizaron dos capas con información geográfica (Área protegida y Huella humana). Los resultados indican que la capa de áreas protegidas tiene mayor influencia que la huella humana. Existe una extensa área de distribución potencial para Dipteryx panamensis, con excelentes áreas en los municipios de El Castillo, Bluefields, y parte de Laguna de Perlas.

El mapa (figura No. 4) refleja áreas extensas de hábitat potencial en áreas protegidas como El refugio de vida silvestre Río San Juan, Reserva Biológica Indio Maíz, Reserva Natural Punta Gorda, Reserva Natural Cerro Silva, Parque Municipal Mahogany y Reserva Natural Wawashang. Según el modelo, las áreas verdes son las de mayor potencial y las azules son hábitat menos viables.

Es necesario recalcar que este modelo no toma en consideración la información climática, por tanto estas áreas podrían ajustarse. Sin embargo, los resultados de este modelo podrían servir para incluir estas zonas (áreas de color verde) en algún plan de recuperación de la especie dentro de áreas protegidas. 


\section{Modelo 2}

Con una combinación de cuatro capas con información bioclimática (precipitación estacional y anual, temperatura anual y general), se corrió el segundo modelo, para probar la influencia del clima sobre el hábitat para la especie Dipteryx panamensis.

Los colores entre amarillo y verde son las zonas más viables para distribución y hábitat de almendro en la zona de estudio.

Este modelo confirma los resultados de densidades de almendros que se encontraron en las comunidades, ya que comparando el mapa (Figura No. 5) con los resultados de densidades (Figura No. 2) se puede ver que la zona de Monkey Point y Bankukuk son poco viables y no precisamente por la huella humana de la zona, sino por condiciones climáticas, esto implica que en las zonas amarillas hay mejores condiciones que en las demás.

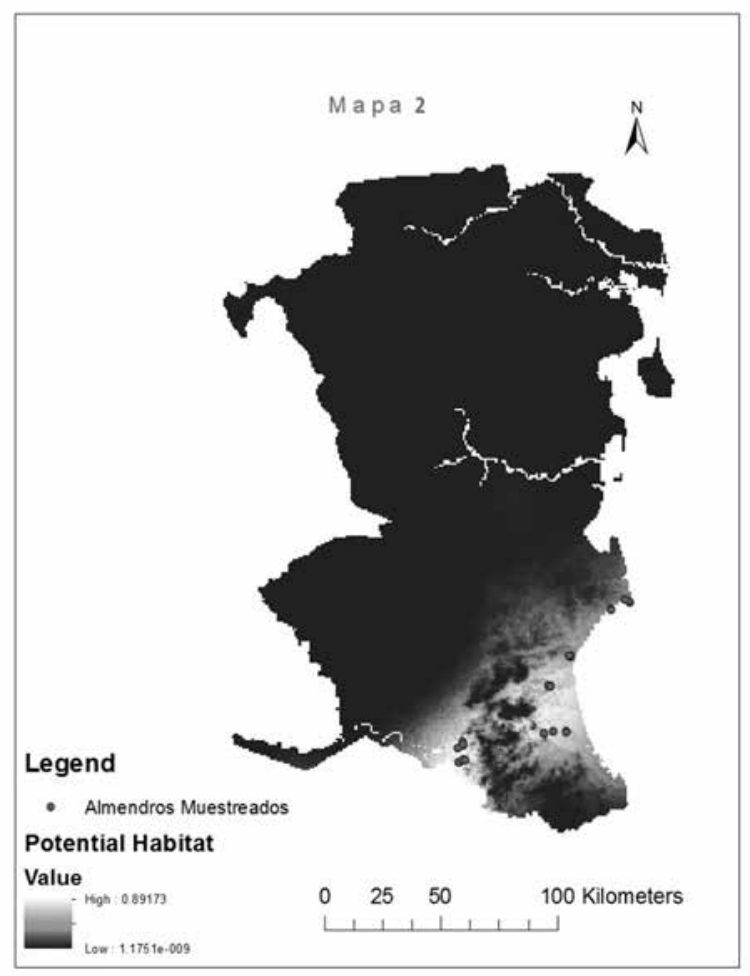

Figura No. 5. Distribución de hábitat potencial del almendro, tomando variables con información climática (Temperatura y Precipitación).

Es importante mencionar que los suelos del sureste de Nicaragua cuentan con las condiciones edáficas adecuadas para el establecimiento del D. panamensis; sin embargo, el incremento del índice de huella humana puede variar las condiciones climáticas, por tal razón, este modelo (el cual cuenta únicamente con variables climáticas) presenta una reducción de zonas viables cerca de las comunidades de Bangkukuk y Monkey Point. Probablemente, los cambios de uso de suelo (potreros) que están experimentando esas comunidades han variado las condiciones climáticas, comparadas con la mayoría de los puntos georeferenciados y utilizados como principal fuente de información en el modelo.

Lo anterior podría indicar que si se logra recuperar las poblaciones o densidad original de almendros, probablemente se podría recuperar las poblaciones de lapas que relatan los pobladores, hubo en sus comunidades antes de la llegada de los colonos y el paso del huracán Joan en 1988. 


\section{Conclusiones}

En las comunidades del sureste de Nicaragua es posible encontrar poblaciones de almendros con densidades promedias de 4.4 árboles $/ \mathrm{Ha}$ (con DAP $\geq 70 \mathrm{~cm}$ ) en las zonas con mayor potencial, aunque es probable que esta densidad varíe con un muestreo más intenso.

Es preciso realizar estudios sobre la regeneración natural del almendro en la zona de estudio, para comprender mejor el comportamiento de sus densidades y tomar decisiones respecto a los usos que le dan los pobladores.

Los modelos de máxima entropía de distribución de especies muestran las zonas del Caribe nicaragüense, donde se podría intensificar las acciones para promover la conservación del hábitat del almendro y recuperar las zonas que han sido degradadas.

Existe alto potencial tanto para el hábitat del almendro así como para la lapa verde, sí y sólo sí se mantienen los esfuerzos institucionales en la zona de amortiguamiento de la reserva biológica Indio Maíz y si en el Caribe se logra mitigar (con la demarcación y saneamiento del territorio Rama y Kriol) el avance de los colonos hacia las áreas protegidas.

Las comunidades de Point of Rock, Corn River e Indian River son comunidades con altos potenciales, ya que hay densidades y biodiversidad forestal altas y son comunidades con poblaciones humanas bajas. También Bartola y la Bijagua presentan altos potenciales para la conservación del hábitat de la lapa verde, empero, de descontinuar los esfuerzos institucionales existentes en la zona, estas comunidades perderían potencial en el largo plazo, por la gran cantidad de pobladores y el uso insostenible que le dan al almendro.

Específicamente en las comunidades de El Castillo, donde la mayoría de sus pobladores son mestizos, es necesario fomentar acciones para reducir las quemas en los potreros y bosques y alguna alternativa que ayude a mitigar el uso del almendro como combustible para cocinar.

Es de vital importancia continuar impulsando investigaciones científicas sobre estas especies o sobre otras especies claves dentro de esta zona de estudio, así mismo acciones en pro de la conservación del medio ambiente en las comunidades del sureste de Nicaragua, una zona tan diversa biológica y etnográfica. 


\section{Lista de referencias}

CITES (2010). Listados Actualizados de las Especies de Fauna y Flora Incluidas en los apéndices de la CITES, distribuidas en Centroamérica y República Dominicana, Comisión Centroamericana de Ambiente y Desarrollo (CCAD) Centroamérica, Pp 27,45

García, M., N. Estrada, and CA Jordan (2012). In Review. Connectivity in the Mesoamerican Biological Corridor: An Assessment of Potential Baird's Tapir Habitat in Northern Central America. Centroamérica.

Madriz V. Bernardo (2004). Relación de dependencia directa para la alimentación y anidación de la lapa verde (Ara ambigua) y el almendro (Dipteryx panamensis) en la zona norte de Costa Rica. Costa Rica.

MARENA (2006). Resolución Ministerial No. 029-2006, Incluir en el sistema de vedas de especies silvestres Nicaragüenses en el listado de vedas nacionales indefinidas a la especie almendro Dipteryx panamensis, La Gaceta No. 141. Managua, Nicaragua.

Phillips Steven J., Anderson Robert P., Schapire Robert E. (2006). Maximum entropy modeling of species geographic distributions. Ecological Modelling 19o pp231-259.

Riverstone Gerald (2008). La Tierra de Nuestros Ancestros, Territorio Rama y Creole en el Caribe Nicaragüense. Managua.

Ruiz Antonio \& Mariscal Teresa, Chassot Olivier \& Monge Guisselle, (2005). El Almendro Un albergue de vida en el Bosque. Nicaragua-Costa Rica, Pp 15

Sanchez, PE; Flores, EM. (1992). Dypterix panamensis, Stryphno dendronexcelsum, Virola koschnyi. Árboles y Semillas del Neotropico. Vol. 1, No. 1. Museo Nacional de Costa Rica, Herbario Nacional de Costa Rica; San José; Costa Rica.

Stiles, G. \& Skutch, A. (1995). Guía de Aves de Costa Rica. Trad. L. Roselli. Ilus. D. 